\title{
A sideways glance. Alcoholic breakdown of barriers: how ethanol can initiate a landslide towards disease
}

\author{
Yula Sambuy
}

Received: 12 February 2009/Accepted: 2 March 2009/Published online: 21 March 2009

(C) Springer-Verlag 2009

Keywords Ethanol - Tight junctions · Intestine · Blood brain barrier . Alcoholic liver disease .

Colorectal cancer

Tight junctions (TJs) are complex and highly regulated morphological structures in areas of close contact between the plasma membrane of neighboring epithelial and endothelial cells. The involvement of TJs in the pathogenesis of a large number of diseases has increasingly been suggested [7]. This is because the barrier role of TJs is essential to guarantee the physical separation and the regulated exchange of substances between the internal and the external environment in many different organs, and a breakdown of this system can allow uncontrolled entry of potentially harmful agents and propagate noxious effects to distant sites in the body. In addition, in recent years a new role for TJs has emerged that links them to the intracellular signaling mechanisms that guide cell proliferation, differentiation and the development of polarity [2]. Thus, TJs have acquired a central role in the control of developmental processes and in the maintenance of normal mucosal homeostasis.

The focus of this sideways glance is on the recently described effects of alterations of TJs permeability by toxic agents in the intestinal mucosa and in the blood brain barrier (BBB) on the onset and progression of various pathologies. In particular, several lines of research have focussed on ethanol due to the increased frequency of

Y. Sambuy $(\bowtie)$

National Research Institute on Food and Nutrition (INRAN),

Via Ardeatina 546, 00178 Rome, Italy

e-mail:sayula@tiscali.it colorectal carcinomas and high incidence of alcoholic liver disease (ALD) in chronic ethanol consumers [5, 11]. In addition, alcohol consumption is a major risk factor in other pathological conditions such as neurobehavioral diseases, inflammation disorders in different organs and enhanced susceptibility to bacterial infections, frequently involving changes in junction permeability of epithelial and endothelial cells accompanied by a strong inflammatory response.

TJs are made up of several transmembrane proteins (claudins, occludin, JAMs, tricellulin) and of the cytoplasmic plaque, a complex network of adaptors and scaffolding proteins that crosslink junctional membrane proteins to the actin cytoskeleton (i.e. ZO-1/2/3, cingulin, PATJ, PAR-3, PAR-6 etc.) as well as of different signaling components such as GTP-binding proteins, protein kinases and post-transcriptional regulators [3]. Immediately below the most apically located TJs, adherens junctions (AJs) consist of two basic adhesive units: the cadherin/catenin and nectin/afadin complexes that play important roles in stabilizing the epithelium by promoting TJ formation and epithelial cell polarity. Cadherins and nectin act as transmembrane adhesion receptors, while the catenins and afadin bind to the actin cytoskeleton and to several regulatory proteins. In particular the catenins (i.e. p120-catenin, $\alpha$-catenin and $\beta$-catenin), locally regulate the organization of the actin cytoskeleton [13], cadherin stability and intracellular signaling pathways involved in the control of gene transcription. Thus, although AJs do not form a physical barrier to macromolecules, they indirectly regulate the integrity of TJs and the epithelial barrier function. A schematic representation of TJs and AJs is shown in Fig. 1. Some excellent reviews have been published on the subject to which the reader is referred for in depth information $[1,3,6,13,21]$. 
The first evidence linking ethanol to changes in epithelial TJs came from a study on cultured intestinal epithelial cells in which cytoskeletal disorganization and increase in paracellular permeability were observed following ethanol treatment. Such changes were shown to result from the activation of myosin light chain kinase (MLCK) that phosphorylates serine residues in myosin 2 light chain, leading to contraction of the perijunctional actomyosin ring and to the opening of paracellular space [10]. The toxic effects of ethanol are generally attributed to the production of acetaldehyde by specific isoforms of alcohol dehydrogenase (ADH) in epithelial and endothelial cells and in the intestinal lumen by the action of enteric microbial fermentation. Acetaldehyde is a highly mutagenic and carcinogenic molecule, also reported to generate a number of reactive oxygen metabolites. At the level of the intestinal epithelium, acetaldehyde has been shown to disrupt TJs and to increase paracellular permeability by different mechanisms (Fig. 2). In a human intestinal Caco2 cell line that lacks ADH activity, acetaldehyde treatment induced inhibition and dissociation of Protein Tyrosine Phosphatase 1 B (PTP1B) from the E-cadherin- $\beta$-catenin complex of the AJ, resulting in sustained tyrosine phosphorylation of $\beta$-catenin and its dissociation from E-cadherin and to loss of homophilic interactions between the extracellular domains of E-cadherin [18]. Since the E-cadherin- $\beta$-catenin complex functions as a suppressor of carcinogenesis and tumor invasion, loss of this interaction may play an important role in the mechanism of acetaldehyde-induced promotion of carcinogenesis and tumor metastasis. Phosphorylated $\beta$-catenin may either undergo degradation by the ubiquitin system or, through the Wnt- $\beta$ -

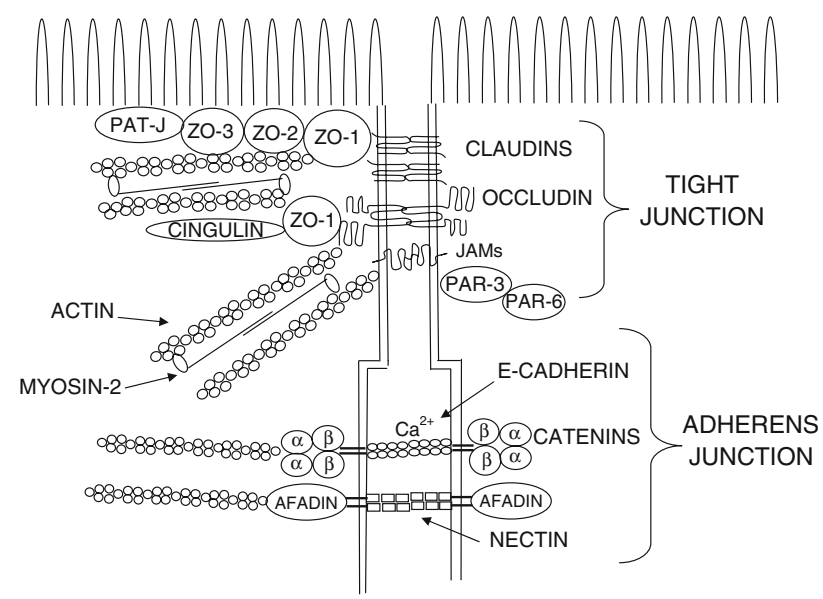

Fig. 1 Tight and adherens junctions composition. A schematic drawing of the composition of tight and adherens junctions. Only the major proteins and their interactions are shown, while, for the sake of clarity, several regulatory proteins participating in the control of junction assembly and function have been omitted. Modified from [17] catenin pathway, migrate to the nucleus and activate the $\beta$-catenin-TCF-4 transcriptional program leading to the expression of genes involved in proliferative events [12]. Inhibition of PTP1B was also shown to block occludin in its phosphorylated state causing its redistribution to the cytoplasm together with ZO-1 [4]. The combined effects of acetaldehyde on the phosphorylation of proteins of TJs and AJs may thus result in increased epithelial barrier permeability and enhanced proliferation, contributing to the development of cancers.

However, acetaldehyde is not the only toxic mediator of ethanol effects (Fig. 2). Recently, chronic low-dose ethanol exposure was shown both in vivo and in vitro to be associated with increased production of phosphatidylethanol through the enzymatic activity of phospholipase D (PLD) in intestinal epithelial cells, resulting in changes in the localization of the TJ proteins claudin 1 and $\mathrm{ZO}-1$ and of the ZO-1-associated transcription factor $\mathrm{ZONAB}$, even in the absence of marked changes to TJs permeability [15]. ZONAB is a Y-box transcription factor [22] that shuttles between the TJs (where it binds to the scaffolding protein $\mathrm{ZO}-1$ ), and the nucleus (where it participates in the regulation of gene expression). Nuclear localization of ZONAB is regulated by the expression levels of $\mathrm{ZO}-1$, which are in turn regulated by cell density. Low density proliferating epithelial cells in culture were shown to express low levels of ZO-1 and high levels of ZONAB accumulating in the nucleus and in TJs. Conversely, high density post-mitotic cells expressed high levels of ZO-1 that sequestered the low levels of ZONAB at the junction [15]. ZO-1 and $\mathrm{ZONAB}$ are involved in the control of cell proliferation by at least two mechanisms: ZONAB interaction with CDK4, a regulator of G1/S phase transition which also co-localizes with ZO-1 at TJs, and direct regulation of cell cycle genes by ZONAB acting as transcription factor [2]. Phosphatidylethanol accumulation following chronic ethanol treatment of intestinal Caco-2 cells was shown to produce detachment of ZONAB from the TJ protein ZO-1 and its migration to the nucleus, resulting in transcriptional activation of genes involved in the initiation of cell proliferation. In addition, phosphatidylethanol accumulation and mislocalization of ZO- 1 and ZONAB were confirmed both in colonic mucosa of ethanol-fed rats and in human colon adenomas of chronic alcohol-consumers, whereas adenomas from non-drinkers did not exhibit such changes [15]. Therefore, the disruption of ZONAB-mediated physiological signals originating from TJ complexes to stop proliferation of confluent epithelial cells might, in addition, facilitate the stimulatory role of other ethanol metabolites, such as acetaldehyde, on the proliferation of cells within intestinal crypts, thereby contributing to the well established co-carcinogenic role of alcohol consumption in the colon-rectum. 


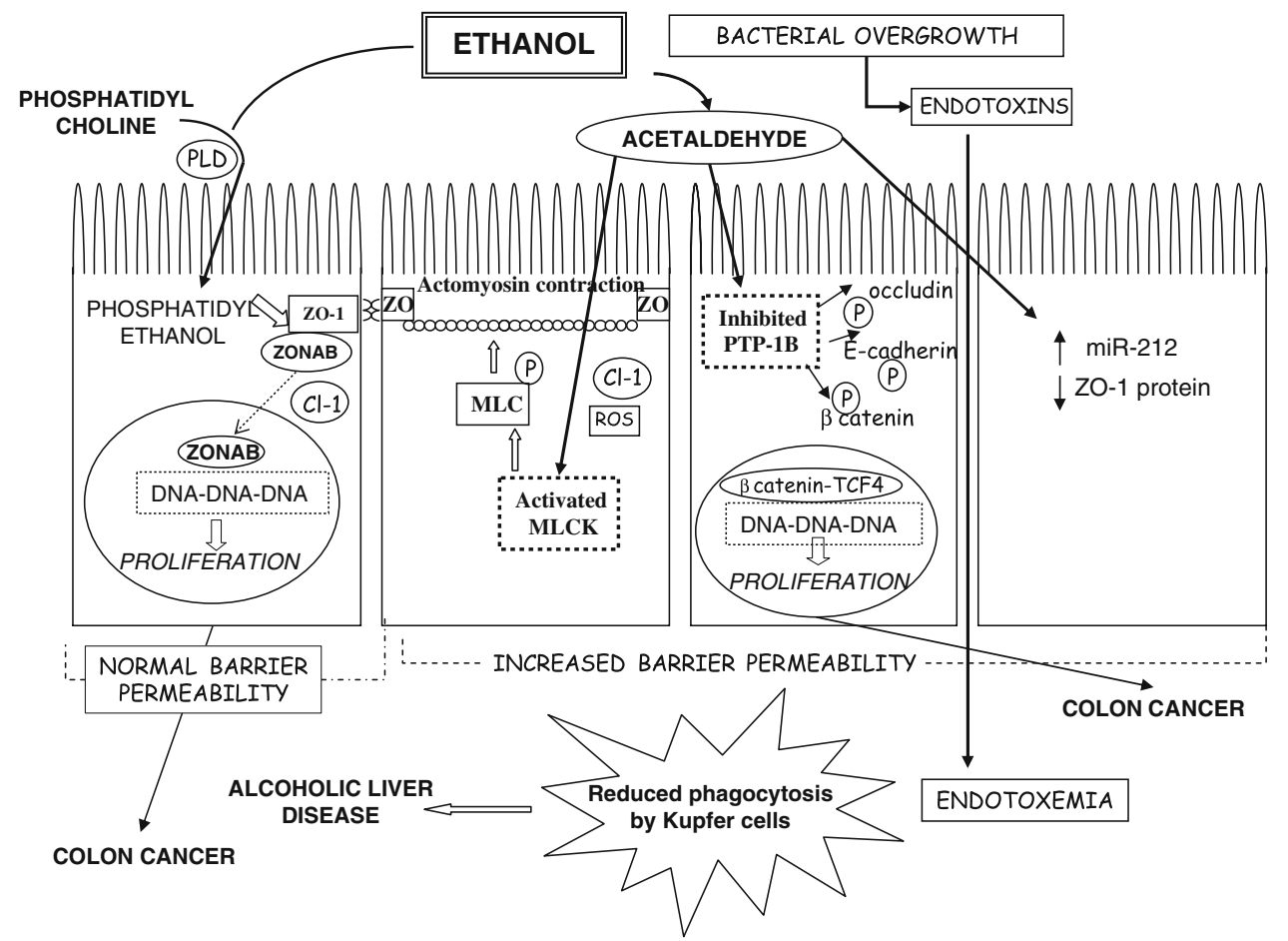

Fig. 2 Multiple pathways of ethanol toxicity in the intestinal epithelium leading to diverse pathological effects. Formation of phosphatidylethanol from ethanol and phosphatidylcholine by the membrane enzyme phospholipase D (PLD) can cause dissociation of the transcription factor $\mathrm{ZONAB}$ from $\mathrm{ZO}-1$ and its translocation to the nucleus, leading to the activation of genes involved in re-initiation of proliferation. These effects occur in the absence of changes in intestinal barrier function. Acetaldehyde, produced in intestinal cells by the enzyme alcohol dehydrogenase can activate phosphorylation of myosin light chain kinase (MLCK), either directly or through the formation of reactive oxygen species (ROS), that in turn phosphorylates myosin 2 light chain (MLC) and causes the contraction of the perijunctional actomyosin ring and increases epithelial barrier permeability, with claudin 1 (Cl-1) internalization. Acetaldehyde can also inhibit the protein tyrosine phosphatase 1B (PTP-1B), blocking

A novel mechanism of regulation of proteins involved in TJ maintenance has been proposed to involve microRNAs. It was recently shown that treatment of human intestinal Caco-2 cells with ethanol under conditions that increase TJs permeability, led to over-expression of the microRNA miR-212 and to decreased expression of ZO-1 protein levels, while the mRNA levels were unaffected [20]. It thus appears that ethanol-induced miR-212 expression mediates inhibition of ZO-1 translation, although not affecting transcription, a mechanism already proposed for other miRNAs [14]. To substantiate these findings the authors found that sigmoid colon biopsies of ALD patients that exhibit increased intestinal permeability, also expressed higher levels of miR-212 and lower amounts of ZO-1 protein compared to healthy controls [20]. This is an interesting novel mechanism of $\mathrm{TJ}$ protein regulation by occludin, $\beta$-catenin and E-cadherin in their phosphorylated state. Phosphorylated occludin is internalized and contributes to barrier dysfunction. Phosphorylated $\beta$-catenin dissociates from E-cadherin and migrates to the nucleus where, with the transcription factor TCF4 , activates genes involved in proliferation. Ethanol also induces down-regulation of the ZO-1 protein as a consequence of the upregulation of miR-212 and this contributes to TJs opening. Chronic ethanol consumption can enhance intestinal bacterial overgrowth with increased production of endotoxins that can freely migrate across the permeabilized intestinal mucosa, leading to endotoxemia. In the liver, acetaldehyde reduces endotoxins clearance by Kupfer cells and can contribute to alcoholic liver disease $(\mathrm{ADH})$. Activation of proliferative events by different ethanol metabolites can contribute to the development of colon carcinogenesis. Modified from [17]

microRNAs that awaits further investigation to elucidate its role in normal physiological conditions.

Chronic or acute ethanol exposure have therefore been shown to cause, by different metabolites and mechanisms of action, breakdown of the barrier function maintained by the apical junctional complex of enterocytes, and to stimulate a proliferative response in post-mitotic differentiated cells.

Elevated intestinal permeability also appears to be one of the major factors in the mechanism of alcohol-induced endotoxemia, a crucial event in the pathogenesis of ALD $[11,16]$. Endotoxins (lipopolysaccharides from the intestinal wall of gram-negative bacteria) derived from the intestinal microflora normally penetrate the mucosa only in trace amounts. In chronic ethanol consumption, microbial proliferation is increased and the acetaldehyde-mediated 
opening of intestinal TJs can significantly enhance the passage of endotoxins into the circulation. Plasma endotoxin levels are higher in patients with ALD than in normal subjects or in patients with non-alcoholic cirrhosis. In addition, alcohol metabolites appear to reduce the phagocytic activity of Kupffer cells, while endotoxins activate cytokine production, further contributing to the development of liver disease. The role of intestinal permeability and endotoxemia in ALD has been reviewed by [16].

Millions of alcoholics exhibit neuro-cognitive defects and neuronal injury associated with degeneration. Although oxidative stress and mitochondrial damage are implicated in tissue injury, the underlying mechanisms of alcoholinduced neurological disorders remain elusive. In cultured brain microvascular endothelial cells (BMVEC), an in vitro model of the BBB, ethanol-activated MLCK led to phosphorylation of serine residues of claudin- 5 and occludin, which resulted in BBB impairment [8]. In addition, ethanol exposure significantly increased the levels and activity of $\mathrm{ADH}$, producing toxic acetaldehyde, and of the EtOHmetabolizing enzyme cytochrome P450-2E1 (CYP2E1) that enhances reactive oxygen species (ROS) generation, implicating oxidative damage in ethanol-induced BBB damage [9]. The in vivo effects of chronic ethanol drinking on the BBB were also studied in rat brain endothelial cells and neurons challenged with the bacterial endotoxin LPS. Increase in endothelial $\mathrm{TJ}$ permeability correlated with decreased mRNA expression and increased phosphorylation of junctional proteins, associated with increased levels of ERK, p38 MAPK, JNK (kinases involved in TJs regulation), and activation of RelA-p50 and p50-p50 (inducers of pro-inflammatory signals). Thus, chronic alcohol drinking may cause higher predisposition to infection and inflammation-related diseases possibly by augmenting the effects of pathogens on the BBB [19]. Current evidence indicates that ethanol-induced changes to the barrier function of the BBB can accelerate pathological changes in the nervous tissue, thus possibly contributing to the onset of different neurocognitive disorders.

Overall, these findings have been obtained in different in vitro models and the only in vivo confirmatory observations were obtained from tissue of chronic alcohol-consumers or of ALD patients, presumably with a long-history of heavy and repeated exposure to high doses of ethanol. However, TJs of the intestinal epithelium are the target of several different noxious agents, including drugs, dietary contaminants or microbial agents, either directly or as a consequence of an inflammatory response. Thus, additive dietary effects could enhance the toxicity of ethanol even at relatively low but repeated dosage. Despite still insufficient in vivo data on possible noxious synergies, a large amount of evidence points to the critical role of TJs and their associated regulatory proteins in mediating the wide arrays of toxic effects of ethanol, showing that breakdown of epithelial and endothelial barriers can initiate a chain of events contributing to the pathophysiology of very different diseases throughout the body.

\section{References}

1. Anderson JM, Van Itallie CM (2008) Tight junctions. Curr Biol 18:R941-R943

2. Balda MS, Matter K (2009) Tight junctions and the regulation of gene expression. Biochim Biophys Acta. doi:10.1016/j.bbamem. 2008.11.024

3. Balda MS, Matter K (2008) Tight junctions at a glance. J Cell Sci 121:3677-3682

4. Basuroy S, Sheth P, Mansbach CM et al (2005) Acetaldehyde disrupts tight junctions and adherens junctions in human colonic mucosa: protection by EGF and L-glutamine. Am J Physiol Gastrointest Liver Physiol 289:G367-G375

5. Boffetta P, Hashibe M (2006) Alcohol and cancer. Lancet Oncol 7:149-156

6. Ebnet K (2008) Organization of multiprotein complexes at cellcell junctions. Histochem Cell Biol 130:1-20

7. Forster $\mathrm{C}$ (2008) Tight junctions and the modulation of barrier function in disease. Histochem Cell Biol 130:55-70

8. Haorah J, Heilman D, Knipe B et al (2005) Ethanol-induced activation of myosin light chain kinase leads to dysfunction of tight junctions and blood-brain barrier compromise. Alcohol Clin Exp Res 29:999-1009

9. Haorah J, Knipe B, Leibhart J et al (2005) Alcohol-induced oxidative stress in brain endothelial cells causes blood-brain barrier dysfunction. J Leukoc Biol 78:1223-1232

10. Ma TY, Nguyen D, Bui V et al (1999) Ethanol modulation of intestinal epithelial tight junction barrier. Am J Physiol Gastrointest Liver Physiol 276:G965-G974

11. Nagata K, Suzuki H, Sakaguchi S (2007) Common pathogenic mechanism in development progression of liver injury caused by non-alcoholic or alcoholic steatohepatitis. J Toxicol Sci 32:453468

12. Nelson WJ, Nusse R (2004) Convergence of Wnt, beta-catenin, and cadherin pathways. Science 303:1483-1487

13. Niessen CM, Gottardi CJ (2008) Molecular components of the adherens junction. Biochim Biophys Acta 1778:562-571

14. Olsen PH, Ambros V (1999) The lin-4 regulatory RNA controls developmental timing in Caenorhabditis elegans by blocking LIN-14 protein synthesis after the initiation of translation. Dev Biol 216:671-680

15. Pannequin J, Delaunay N, Darido C et al (2007) Phosphatidylethanol accumulation promotes intestinal hyperplasia by inducing ZONAB-mediated cell density increase in response to chronic ethanol exposure. Mol Cancer Res 5:1147-1157

16. Rao RK (2008) Acetaldehyde-induced barrier disruption and paracellular permeability in Caco- 2 cell monolayer. Methods Mol Biol 447:171-183

17. Sambuy Y (in press) Tight junctions as mediators of adverse effects. In: Ballantyne B, Marrs T, Syversen T (eds) General and applied toxicology, 3rd edn. Wiley, London

18. Sheth P, Seth A, Atkinson KJ et al (2007) Acetaldehyde dissociates the PTP1B-E-cadherin-beta-catenin complex in Caco-2 cell monolayers by a phosphorylation-dependent mechanism. Biochem J 402:291-300

19. Singh AK, Jiang Y, Gupta S et al (2007) Effects of chronic ethanol drinking on the blood brain barrier and ensuing neuronal 
toxicity in alcohol-preferring rats subjected to intraperitoneal LPS injection. Alcohol 42:385-399

20. Tang Y, Banan A, Forsyth CB et al (2008) Effect of alcohol on miR-212 expression in intestinal epithelial cells and its potential role in alcoholic liver disease. Alcohol Clin Exp Res 32:355364
21. Tsukita S, Yamazaki Y, Katsuno T et al (2008) Tight junctionbased epithelial microenvironment and cell proliferation. Oncogene 27:6930-6938

22. Wilkinson MF, Shyu AB (2001) Multifunctional regulatory proteins that control gene expression in both the nucleus and the cytoplasm. Bioessays 23:775-787 